\title{
Genetic analysis of an orbital metastasis from a primary hepatic neuroendocrine carcinoma
}

\author{
JACOB Ø. RASMUSSEN ${ }^{1}$, SARAH L. VON HOLSTEIN ${ }^{1,2}$, JAN U. PRAUSE ${ }^{1}$, BEN VAINER $^{3}$, \\ ALASTAIR B. HANSEN ${ }^{4}$, ANDRÉ FEHR ${ }^{5}$, GÖRAN STENMAN ${ }^{5}$ and STEFFEN HEEGAARD ${ }^{1,2}$ \\ ${ }^{1}$ Eye Pathology Institute, Department of Neuroscience and Pharmacology, University of Copenhagen; \\ ${ }^{2}$ Department of Ophthalmology, Glostrup Hospital, University of Copenhagen; ${ }^{3}$ Department of Pathology, \\ Rigshospitalet, University of Copenhagen, Copenhagen; ${ }^{4}$ Department of Pathology, Herlev Hospital, \\ University of Copenhagen, Herlev, Denmark; ${ }^{5}$ Sahlgrenska Cancer Center, \\ Department of Pathology, University of Gothenburg, Gothenburg, Sweden
}

Received May 7, 2014; Accepted June 16, 2014

DOI: 10.3892/or.2014.3345

\begin{abstract}
A 71-year-old female with a known history of primary hepatic neuroendocrine carcinoma, presented with a visual defect, proptosis and restricted eye movements of the right eye. Biopsies from the orbit and from the primary hepatic neuroendocrine carcinoma showed similar morphological and immunohistochemical features, and high-resolution, arraybased comparative genomic hybridization demonstrated loss of one copy each of chromosomes 3 and 18, and gain of 1q both in the primary hepatic neuroendocrine carcinoma and in the orbital tumour. The orbital mass was diagnosed as a metastasis from the primary hepatic neuroendocrine carcinoma. Primary hepatic neuroendocrine tumours are extremely rare, and the orbit is an extremely rare location for a neuroendocrine carcinoma metastasis. This is the first reported case of an orbital metastasis with origin from a primary hepatic neuroendocrine carcinoma.
\end{abstract}

\section{Introduction}

Neuroendocrine tumours (NETs) are slowly growing tumours arising from neuroendocrine enterochromaffin (Kulchitsky) cells, accounting for only $0.66 \%$ of all malignancies. NETs have an increasing incidence and are most commonly found in the gastrointestinal tract $(55 \%)$ followed by the bronchopulmonary system (30\%) (1). NETs are traditionally divided into subgroups based primarily on the anatomical location from where they originate, i.e. the foregut (bronchus, lung, stomach, pancreas, liver and first part of the duodenum), the midgut

Correspondence to: Professor Steffen Heegaard, Department of Neuroscience and Pharmacology, Eye Pathology Institute, University of Copenhagen, Frederik V's vej 11, DK-2100 Copenhagen, Denmark E-mail: sthe@sund.ku.dk

Key words: primary hepatic neuroendocrine carcinoma, carcinoid, orbital metastasis, arrayCGH analysis (second part of the duodenum, jejenum, ileum, appendix and proximal colon) and the hindgut (distal colon, rectum and genitourinary tract) $(2,3)$. However, since the tumours in each group do not always share similar clinical behaviour a new classification system is needed for the future.

NETs most commonly metastasise to lymph nodes, liver, lungs, peritoneum and pancreas (4). There is a tendency that orbital metastases originate from primary gastrointestinal tract NETs, whereas intraocular metastases arise from primary bronchial NETs (5). Primary hepatic NETs are extremely rare, and orbital metastases from NETs in general have been reported only in a very few cases (5-9). We present the first case of a primary hepatic NET with an orbital metastasis including the genomic profiles of the two tumours.

\section{Patients and methods}

Case report. A 71-year-old female presented with impaired vision and a visual field defect of her right eye. Six years earlier she had been diagnosed with a large primary hepatic NET weighing $5.5 \mathrm{~kg}$ and having the greatest diameter of $35 \mathrm{~cm}$. The tumour was resected surgically (Fig. 1A).

Visual acuity at referral was 0.3 of the right eye and 0.8 of the left eye. A 2-mm protrusion of the right eye as well as restricted ocular motility at superior and temporal gaze were observed. Furthermore, a visual field defect was present in the periphery of all 4 quadrants. Computed tomography scan (CT) and contrast enhanced magnetic resonance imaging (MRI) scan demonstrated a retrobulbar, intraconal mass measuring $2.2 \times 1.8 \times 1.5 \mathrm{~cm}$ with contrast uptake (Fig. 2A). Somatostatin receptor scintigraphy (octreoscan) showed increased uptake in the cranium, neck, mediastinum, right upper arm, abdomen and between the right kidney and the liver. A biopsy from the right orbit via the upper eyelid was performed. Ptosis of the right eye was permanently present after the procedure. The patient underwent 30 Gy radiation to the right orbit, but the tumour did not respond. Fifteen months later, the orbital tumour was surgically excised en bloc. It was adherent to the superior rectus muscle and the levator muscle of the eyelid. 


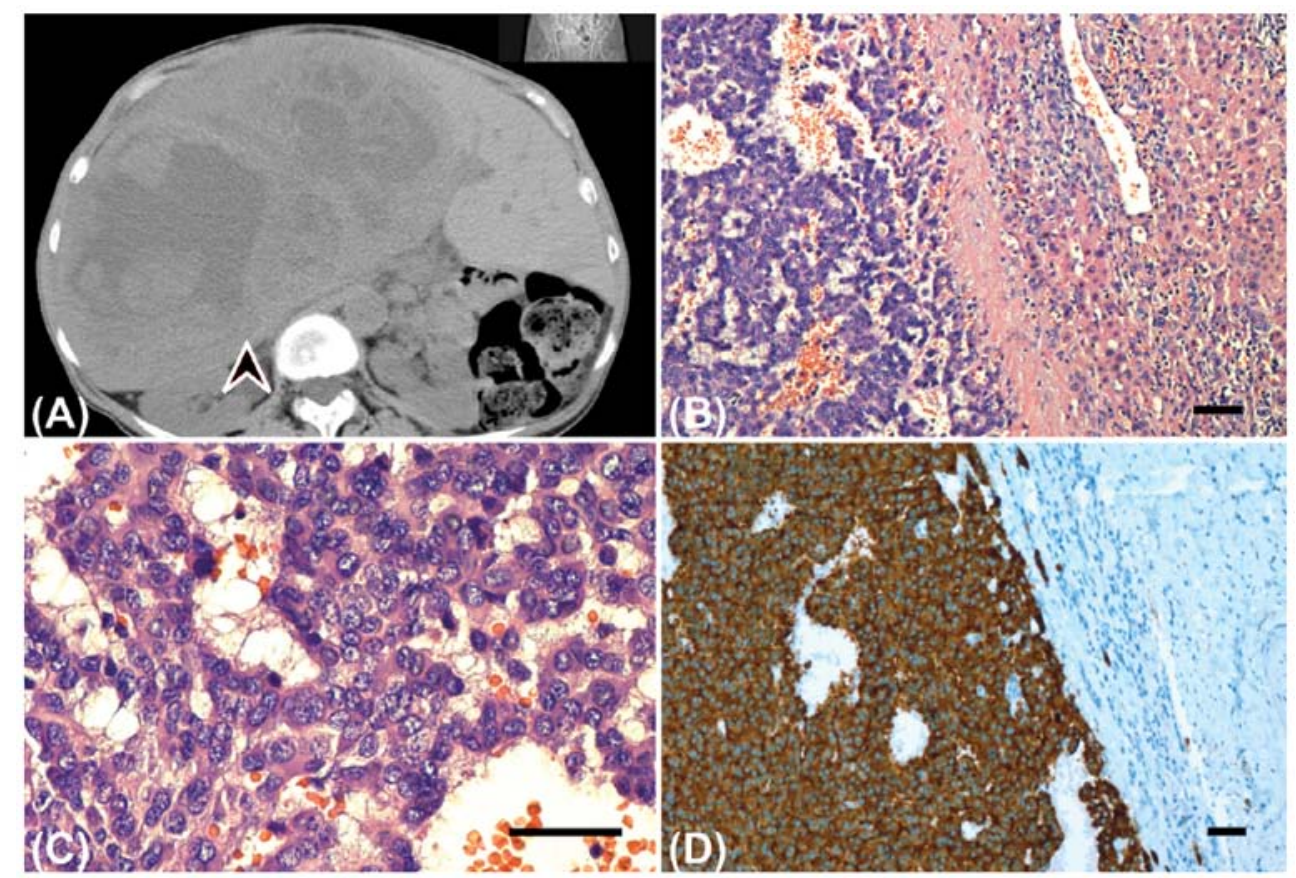

Figure 1. (A) Computed tomography scan revealing a large tumour in the right liver lobe (arrowhead) stretching into the 4th segment of the left liver lobe. (B) The hepatic tumour at the border of the tumour; the tumour cells are to the left of the micrograph. Inflammatory cells are observed outside the tumour. (H\&E). Scale bar, $100 \mu \mathrm{m}$. (C) The hepatic tumour cells are arranged in a trabecular network and consist of relatively uniform tumour cells arranged in anastomosing cell strands (H\&E). Scale bar, $50 \mu \mathrm{m}$. (D) Hepatic tumour cells showing positive immunoreactivity for synaptophysin. Scale bar, $100 \mu \mathrm{m}$.

Vision declined to 0.17 at the right eye. Metastases to the cerebrum developed 20 months later and they were surgically removed. Metastasis to the sacral bone developed 8 years after initial diagnosis of the hepatic neuroendocrine carcinoma and was treated with $20 \mathrm{~Gy}$ radiation. When the neuroendocrine carcinoma had started to spread 8 years after the initial diagnosis, Intron $A^{\circledR}$, Sandostatin ${ }^{\circledR}$, Nexavar ${ }^{\circledR}$ and Dotatoc ${ }^{\circledR}$ were instituted. Twelve years after diagnosis of the primary hepatic neuroendocrine carcinoma, the patient was treated with Temodal, but due to progression of the liver metastases, the treatment was changed to etoposide monotherapy. This was, however, also stopped due to the continuous progression of the liver metastases and increasing renal failure.

At the last follow-up time (15 years after the diagnosis of hepatic NET) an MRI scan revealed a recurrent mass measuring $2 \times 1.5 \times 1.5 \mathrm{~cm}$ in the right orbit. The patient is still alive and receives palliative treatment. She has no light perception in the right eye and only 0.4 in the left eye.

Histopathology. The orbital biopsy and the liver specimen were formalin-fixed and paraffin-embedded (FFPE), and 4- $\mu \mathrm{m}$ sections were mounted on glass slides. The sections were stained with haematoxylin and eosin (H\&E) and periodic acid-Schiff (PAS). Immunohistochemical reactions were performed using the streptavidin-biotin method. Antibodies against CD31, CD34, CD56, pan-cytokeratin (clone AE1/AE3), CK7, chromogranin A, Ki-67 (clone MIB-1), neuron-specific enolase (NSE), S100, serotonin, somatostatin, synaptophysin, thyroid transcription factor (TTF-1) and vimentin (all from Dako Inc., Copenhagen, Denmark) were applied. HepPar-1 antibody, polyclonal antibodies against CEA (both from Dako) and monoclonal antibodies against arginase-1 (Abcam, Cambridge, UK) were applied to rule out that the tumour was a hepatocellular carcinoma with neuroendocrine dedifferentiation. Appropriate controls were performed.

ArrayCGH. Genomic DNA was isolated from the FFPE tumour tissues from the primary tumour and the metastatic lesion using the DNeasy ${ }^{\circledR}$ Blood and Tissue kit (Qiagen $\mathrm{GmbH}$, Hilden, Germany). ArrayCGH analysis was subsequently performed using the human genome $\mathrm{CGH}$ microarray 244K oligonucleotide array (G4411B; Agilent Technologies Inc., Palo Alto, CA, USA). The arrayCGH experiments were performed essentially as previously described and as recommended by the manufacturer $(10,11)$. Slides were scanned on an Agilent High-Resolution C Microarray Scanner, followed by data extraction and normalization using Feature Extraction v.10.7.1 (Agilent Technologies) with linear normalization (protocol CGH_107_Sep09). Data analysis was carried out using Nexus Copy Number Software ${ }^{\circledR}$ Discovery Edition v. 6.0 (BioDiscovery Inc., El Segundo, CA, USA) as previously described (11). Each aberration was checked manually to confirm the accuracy of the call. Regions partially or completely covered by a previously reported copy number variation (Database of Genomic Variants; http://dgvbeta.tcag. ca/dgv/app/home?ref=NCBI36/hg18) were excluded from the analysis.

\section{Results}

Histopathology. A biopsy from the orbital mass contained nests of pleomorphic tumour cells (Fig. 2B), that diffusely infiltrated the orbital connective tissue and fat. The tumour cells were intermediate-sized and polyhedral with eosinophilic cytoplasm with perinuclear eosinophilic inclusions and rounded to oval nuclei with finely granular chromatin. There 


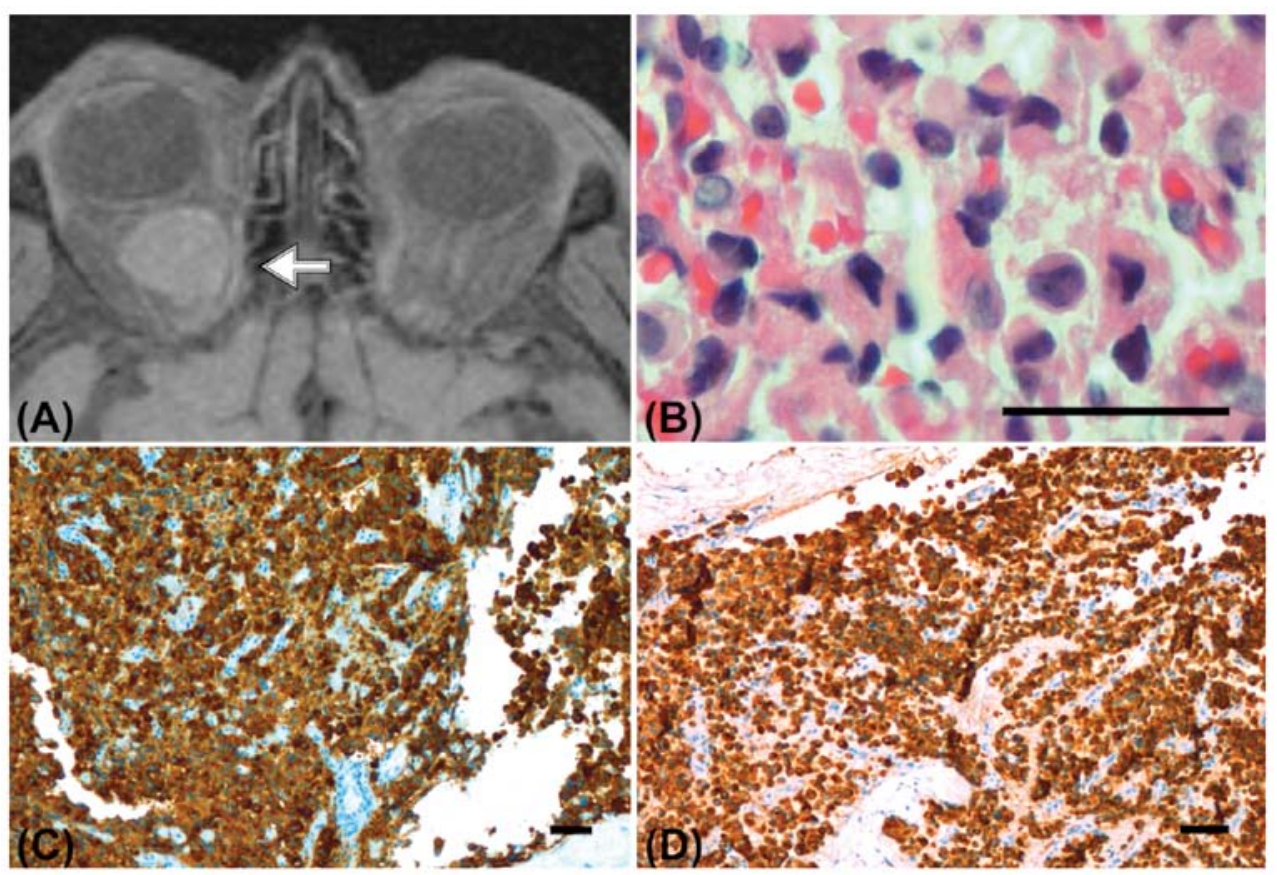

Figure 2. (A) Magnetic resonance imaging scan of the orbit demonstrating a retrobulbar intraconal mass (arrow) measuring $2.2 \times 1.8 \times 1.5 \mathrm{~cm}$ with contrast uptake. (B) The biopsy of the orbital tumour showing pleomorphic tumour cells with eosinophilic cytoplasm and irregular nuclei with granular chromatin (H\&E). Scale bar, $50 \mu \mathrm{m}$. (C) Orbital tumour cells positive for anti-synaptophysin. Scale bar, $100 \mu \mathrm{m}$. (D) Orbital tumour cells showing positive immunoreactivity for anti-pancytokeratin AE1/AE3. Scale bar, $100 \mu \mathrm{m}$.

were no areas of necrosis, and mitoses were infrequent. The orbital tumour cells demonstrated positive immunoreactivity for synaptophysin (Fig. 2C), CD56, cytokeratin (AE1/AE3) (Fig. 2D), vimentin, and NSE. Scattered cells were positive for somatostatin, whereas staining for chromogranin was negative. Stainings for hepatocytes (arginase-1, polyclonal CEA and HepPar1) were negative. The average proliferation index (Ki-67) was $<10 \%$.

The histopatological assessment of the resected liver tumour revealed a trabecularly arranged pattern consisting of relatively uniform cells predominantly placed in anastomosing, irregular cell strands. The stroma was highly vascular. The tumour cells contained round or oval nuclei, abundant pink-coloured and granular cytoplasm, and infrequent mitoses (Fig. 1B and 1C). Immunohistochemistry of the liver tumour was consistent with the immunoprofile of the orbital specimen (Fig. 1D). These findings were in agreement with the diagnosis of a primary low-grade hepatic NET with an orbital metastasis.

Genomic imbalances. Genome-wide arrayCGH analysis of the primary tumour using $244 \mathrm{~K}$ arrays revealed a quiet genome with few copy number alterations. Three major aberrations were detected including gain of one copy of the long arm of chromosome 1 (1q21.1-qter) and losses of one copy each of chromosomes 3 and 18 (Fig. 3). No segmental aberrations, high-copy gains/amplifications or homozygous deletions were detected.

The DNA isolated from the orbital metastasis was degraded and yielded array data of poorer quality. However, analysis of this array using less stringent criteria for aberration calls confirmed the presence of the same aberrations as in the liver tumour as well as gain of 2q24.2-qter and loss of one copy of the long arm of chromosome 10 (data not shown).

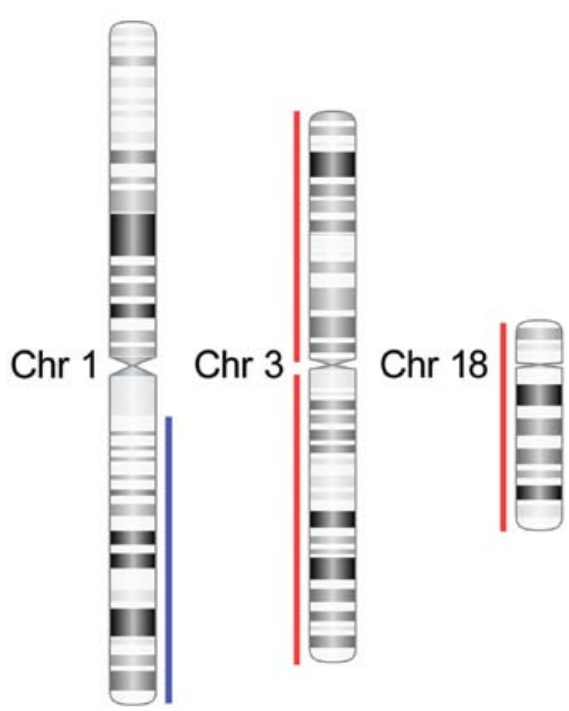

Figure 3. ArrayCGH profiles of the hepatic neuroendocrine carcinoma with copy number alterations involving chromosomes 1,3 and 18. Gains are indicated by vertical blue lines and losses by vertical red lines.

\section{Discussion}

We here present the first reported case of an orbital metastasis with origin from a primary hepatic neuroendocrine carcinoma including genetic analysis of both tumours. Primary hepatic NETs are extremely rare with only $90-100$ cases reported to date (6), and the orbit is an extremely rare anatomical site for metastasis from NETs with less than 70 reported cases in the literature (5,7-9). The 5-year survival rate for hepatic neuroendocrine carcinoma is reported to be only $18 \%$ (2). In 
the present case, the patient is still alive 15 years after being diagnosed with the liver tumour, but only after intense surgical and oncological treatment. The 'golden standard' of treating orbital carcinoid is biopsy followed by local radiotherapy and additional systemic chemotherapy (5). In the present case, the orbital tumour was removed surgically, since the tumour did not respond adequately to radiotherapy and systemic chemotherapy and is also at present showing evidence of local recurrence.

Using arrayCGH we demonstrated that both the primary hepatic NET and the orbital metastasis had gain of one copy of 1q and losses of one copy each of chromosomes 3 and 18 (Fig. 3). Loss of chromosome 18 is a common feature in gastrointestinal NETs and has been reported in $43-88 \%$ of tumours $(12,13)$. Deletion of $18 q 21$, the location of the tumour suppressor genes DCC (deleted in colorectal carcinoma) and DPC4/SMAD4 (involved in transcriptional regulation), may play a role in the pathogenesis of gastrointestinal and pancreatic endocrine tumours (13). Loss of chromosome 3 is only found in approximately $25 \%$ of gastrointestinal NETs (12). In contrast, loss of chromosome $3 p$ has been reported as the most common alteration in NETs of the lung (14). A high frequency of loss of heterozygosity (LOH) at a locus proximal to the von Hippel-Lindau (VHL) gene on $3 p$ has been shown to be necessary for malignant conversion of pancreatic islet cell tumours, and $\mathrm{LOH}$ at $3 \mathrm{q}(15)$ is reported in $50 \%$ of sporadic pancreatic endocrine tumours associated with hepatic metastases (12).

Hepatocellular carcinomas (HCC) metastasising to the orbit are extremely rare and only 17 cases have been reported in the English language literature (16). In Europe, the incidence of $\mathrm{HCC}$ is in the range 3-12 of 100,000 individuals, whereas it exceeds 30 of 100,000 in East Asia (17). Common genetic alterations in HCC are mutations in TP53 and CTNNB1 (the gene for $\beta$-catenin) and amplification of genes in 1q21-q22 (18). In contrast, the observed alterations involving chromosomes 3 and 18 in the present case are uncommon in HCC (18).

Taken together, the present hepatic NET and its orbital metastasis have genetic features consistent with gastrointestinal NETs. The mechanism for development of primary hepatic NETs is unclear to date, while theories include origin from the biliary system or from ectopic adrenal or pancreatic tissues in the liver (19). The results of the present study indicate that hepatic NETs exhibit genetic similarities mainly to gastrointestinal NETs; however, further studies are needed to clarify these issues. Genetic studies may also provide new biomarkers that can form the basis for a new classification system and serve as prognostic biomarkers.

\section{References}

1. Pinchot SN, Holen K, Sippel RS and Chen H: Carcinoid Tumors. Oncologist 13: 1255-1269, 2008.

2. Williams ED and Sandler M: The classification of carcinoid tumours. Lancet 1: 238-239, 1963.

3. Friedman JR and Kaestner KH: On the origin of the liver. J Clin Invest 121: 4630-4633, 2011.

4. Modlin IM, Lye KD and Kidd M: A 5-decade analysis of 13,715 carcinoid tumors. Cancer 97: 934-959, 2003.

5. Mehta JS, Abou-Rayyah Y and Rose GE: Orbital carcinoid metastases. Ophthalmology 113: 466-472, 2006.

6. Fenoglio LM, Severini S, Ferrigno D and Gollè G: Primary hepatic carcinoid: A case report and literature review. World J Gastroenterol 15: 2418-2422, 2009.

7. Turaka K, Mashayekhi A and Shields Cl: A case series of neuroendocrine (carcinoid) tumor metastasis to the orbit. Oman J Ophthalmol 4: 125-128, 2011.

8. Gupta A, Chazen JL and Phillips CD: Carcinoid tumor metastases to the extraocular muscles: MR imaging and CT findings and review of the literature. AJNR Am J Neuroradiol 32: 1208-1211, 2011.

9. Matsuo T, Ichimura K, Tanaka T and Takenaka T: Neuroendocrine tumor (carcinoid) metastatic to orbital extraocular muscle: case report and literature review. Strabismus 18: 123-128, 2010.

10. Barrett MT, Scheffer A, Ben-Dor A, Sampas N, Lipson D, Kincaid R, Tsang P, Curry B, Baird K, Meltzer PS, Yakhini Z, Bruhn L and Laderman S: Comparative genomic hybridization using oligonucleotide microarrays and total genomic DNA. Proc Natl Acad Sci USA 101: 17765-17770, 2004.

11. Persson F, Winnes M, Andrén Y, et al: High-resolution array CGH analysis of salivary gland tumors reveals fusion and amplification of the FGFR1 and PLAG1 genes in ring chromosomes. Oncogene 27: 3072-3080, 2008.

12. Zikusoka MN, Kidd M, Eick G, Latich I and Modlin I M: The molecular genetics of gastroenteropancreatic neuroendocrine tumors. Cancer 104: 2292-2309, 2005.

13. Andersson E, Swärd C, Stenman G, Ahlman H and Nilsson O: High-resolution genomic profiling reveals gain of chromosome 14 as a predictor of poor outcome in ileal carcinoids Endocr Relat Cancer 16: 953-966, 2009

14. Leotlela PD, Jauch A, Holtgreve-Grez H and Thakker RV: Genetics of neuroendocrine and carcinoid tumours. Endocr Relat Cancer 10: 437-450, 2003.

15. Tönnies H, Toliat MR, Ramel C and Pape UF: Analysis of sporadic neuroendocrine tumours of the enteropancreatic system by comparative genomic hybridisation. Gut 48: 536-541, 2001.

16. Guerriero $S$, Infante $G$ and Giancipoli E: Hepatocellular carcinoma metastasis to the orbit in a coinfected $\mathrm{HIV}^{+} \mathrm{HBV}^{+}$ patient previously treated with orthotopic liver transplantation: a case report. Case Rep Ophthalmol Med: 2011: 549270, 2011.

17. Hirunwiwatkul P, Tirakunwichcha S, Meesuaypong $P$ and Shuangshoti S: Orbital metastasis of hepatocellular carcinoma. J Neuroophthalmol 28: 47-50, 2008.

18. van Malenstein H, van Pelt J and Verslype C: Molecular classification of hepatocellular carcinoma anno 2011. Eur J Cancer 47: 1789-1797, 2011.

19. Gao J, Hu Z, Wu J, Bai L and Chai X: Primary hepatic carcinoid tumor. World J Surg Oncol 9: 151, 2011. 